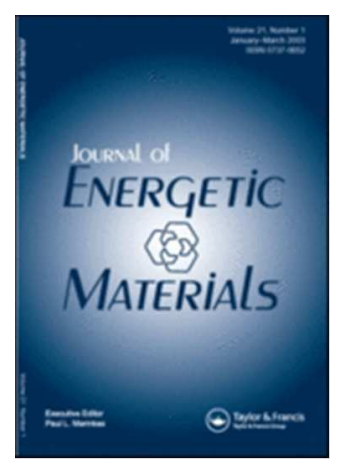

\title{
Azidated Ether-Butadiene-Ether Block Copolymers as Binders for Solid Propellants
}

\begin{tabular}{|r|l|}
\hline Journal: & Journal of Energetic Materials \\
\hline Manuscript ID: & UEGM-2015-1340.R3 \\
\hline Manuscript Type: & Original Article \\
\hline Date Submitted by the Author: & n/a \\
\hline Complete List of Authors: & $\begin{array}{l}\text { Cappello, Miriam; University of Pisa, Civil and Industrial Engineering } \\
\text { Lamia, Pietro; University of Pisa, Civil and Industrial Engineering } \\
\text { Mura, Claudio; University of Pisa, Civil and Industrial Engineering } \\
\text { Polacco, Giovanni; University of Pisa, Civil and Industrial Engineering } \\
\text { Filippi, Sara; University of Pisa, Civil and Industrial Engineering }\end{array}$ \\
\hline Keywords: & Azides, HTPB, block copolymers, energetic binders \\
\hline & \multicolumn{2}{|c|}{} \\
\hline
\end{tabular}

SCHOLARONE ${ }^{\text {Ix }}$

Manuscripts 


\title{
Azidated Ether-Butadiene-Ether Block Copolymers as Binders for Solid Propellants
} Short title: Azidated Ether-Butadiene Energetic Copolymers

\author{
Miriam Cappello, Pietro Lamia, Claudio Mura, Giovanni Polacco*, Sara Filippi \\ Department of Civil and Industrial Engineering \\ University of Pisa \\ Largo Lucio Lazzarino, 1 - 56122 Pisa, ITALY \\ * Corresponding author \\ e-mail: giovanni.polacco@unipi.it \\ phone: +390502217820 \\ fax: +390502217866
}

\begin{abstract}
Polymeric binders for solid propellants are usually based on hydroxyl-terminated polybutadiene (HTPB), which does not contribute to the overall energy output. Azidic polyethers represent an interesting alternative, but may have poorer mechanical properties. Polybutadiene-polyether copolymers may join the advantages of both. Four different etherbutadiene-ether tri-block copolymers were prepared and azidated starting from halogenated and/or tosylated monomers and using HTPB as initiator. The presence of the butadiene block complicates the azidation step and reduces the storage stability of the azidic polymer.

Nevertheless, the procedure allows modifying the binder properties by varying the type and the lengths of the energetic blocks.
\end{abstract}




\section{Introduction}

In the most common propellant formulations, the solid ingredients are mixed with a polymer, which is subsequently cast cured to give an elastomeric network. The polymer binder acts by wetting the solid thus providing a void-free matrix that allows the formulation to be cast into large and irregular cases [1]. The polymer provides mechanical integrity to the final product and, together with a plasticiser, ensures safety during handling, since it absorbs and dissipates energy from hazardous stimuli that may happen during storage and transportation [2]. The most commonly used binder is hydroxy-terminated polybutadiene (HTPB) cross-linked with isocyanates to give a polyurethanic network. Alternatively, carboxy-terminated polybutadiene and hydroxyl-terminated polyethers have been also suggested [2]. HTPB is used worldwide and its success from the mechanical and safety point of view is well known and recognized. However, from the propellant point of view, due to its inert character, there are many cases when it is just a dead weight, which does not contribute to the overall energy output and limits the performance of the composition unless there is a high solids loading. On the other hand, high solids loadings induce processing and vulnerability problems. Therefore, the main goal of binder research is to find a formulation that reduces vulnerability, shock and impact insensitivity without lowering performance. A possible approach is to substitute HTPB with alternative materials, such as the so-called energetic-polymers, that act as binder and at the same time contribute to the output energy. The simplest way to achieve this result is the inclusion of functional groups ( such as nitro $\left(\mathrm{NO}_{2}\right)$, nitrate $\left(\mathrm{NO}_{3}\right)$ or azide $\left(\mathrm{N}_{3}\right)$ ), which may increase the internal enthalpy of formation of the formulation and/or improve the overall oxygen balance. Of course, this has to be done by preserving all the main requirements of a binder, which should [3]:

(a) be a liquid with a good processability at the mixing temperatures $\left(30-60{ }^{\circ} \mathrm{C}\right)$; 
(b) be curable, with minimum evolution of heat at $40-80{ }^{\circ} \mathrm{C}$ and give an elastomeric network with good mechanical properties;

(c) have a glass transition temperature possibly below $-40{ }^{\circ} \mathrm{C}$;

(d) be compatible with all the other ingredients of the formulation.

During the last decades, several energetic binders have been synthesized and here we will mention only the most promising ones, but the interested reader may find detailed information and a much longer list in the reviews by Agrawal [4], Provatas [2], Badgujar et al. [5], Sikder and Sikder [6] and Gaur et al. [7].

The first idea that comes to mind is the introduction of a limited quantity of energetic groups directly into HTPB. The early studies to produce nitrated HTPB (NHTPB) suggested a nitromercuration-demercuration route, [8], while later on Colclough et al. [1] started from epoxidation of HTPB and then reacted $\mathrm{N}_{2} \mathrm{O}_{5}$ with epoxide groups to form dinitrate esters. Of course, the level of nitration affects also thermal stability and mechanical properties and the authors suggested that a nitration corresponding to $10 \%$ of double bonds gives a good compromise between energy output, mechanical properties and miscibility with energetic plasticizers.

Alternatively, the energetic binders may have a chemical structure completely different from HTPB and the most studied one are those containing nitro or azidic groups. In the first case, popular polymers derives from cationic polymerization of 3,3 (nitratemethyl) methyl oxetane 3-nitratomethyl-3-methyl oxetane (NIMMO) and glycidyl nitrate (GLYN). Again, the OHterminated chains are able to crosslink into polyurethane rubbers. By comparing these nitrated polymers, Agrawal [4] suggested that NHTPB has the lower production cost, while the other two have better performances. In the case of azidic functionalities, there is a slightly longer list of candidates, but the most studied one is glycidyl azide polymer (GAP). GAP has been employed also as a plasticizer [4] and several publications may be found concerning its 
synthesis as well as its thermal behavior and explosive properties [9-25]. The GAP synthesis was first described in a patent filed in 1972 by Vandenburg [26] who did azidation of polyepichlorohydrin (PECH) by using sodium azide in dimethylformamide. Twenty years later, Frankel et al. described a semi-industrial production in USA, sponsored by the Air Force Astronautic Laboratory [27]. The PECH was obtained by polymerization of epichlorohydrin $(\mathrm{ECH})$ by using glycerol as the initiator to give a triol polymer easily crosslinkable with isocyanates. In the following decades, the synthetic route has not changed significantly and sodium azide remains the preferred reagent. The azidation can be performed in different organic solvents and in water [27, 28]. In the first case, the reaction is much faster and in dimethyl sulphoxide (DMSO) it is reported to occur at $90-95^{\circ} \mathrm{C}$ within $12-18 \mathrm{~h}$. In water, the use of a phase transfer catalyst is necessary and the reaction may take several days before completion at comparable temperatures. Nevertheless, the two processes give GAP of comparable quality. PECH may be in linear, star or branched form depending on the catalyst, initiator and operating conditions. Consequently, the number of hydroxyl functionalities per chain can be modulated in a wide range of values. Moreover, $\mathrm{PECH}$ is now commercially available as well as GAP. Starting from GAP, several alternative organic azides have been developed in the last years and the use of the azido group is gaining more and more attention since it has also other advantages like i.e. reducing the flame and smoke in exhaust gases, thus making the propellant formulations more eco-friendly [5]. Some examples of suggested polymeric azides are poly(3,3 bis(azidomethyl)oxetane-co- $\varepsilon$-caprolactone) [29], 3,3 bis(azidomethyl)oxetane-tetrahydrofuran [30, 31] and polyglycidylazide- $b$-poly(azidoethyl methacrylate) [32]. Among the proposed ones, the polyoxetanes, first synthesized by Manser [33-35], gained an important role in the field of energetic binders. Manser started from monomers such as 3-nitratomethyl-3-methyl oxetane (NIMMO), 3,3-bis(azidomethyl)oxetane (BAMO) and its analog monofunctional 3-azidomethyl-3-methyl 
oxetane (AMMO). Later on, he also described the synthesis of many different oxetanes with cubyl and carboranyl groups, but the difficult syntheses involved have so far precluded their evaluation in large-scale formulations [1]. In the case of BAMO and AMMO, a chlorinated or tosylated monomeric precursor was azidated and then subjected to cationic ring-opening polymerization (CROP) by using a diol and a Lewis acid as catalyst. The classical Active Chain End (ACE) mechanism proceeds by donation of a proton from initiator to the oxetane, which then propagates with more oxetane monomers until the chain is terminated either with water or alcohol to give the hydroxy-terminated polymer. The number of hydroxyl functionalities per chain should theoretically coincide with those of the alcohol used as initiator.

The molecular weight can be adjusted by changing monomer feed rates and the ratio of diol to Lewis acid, but of course a post-polymerization chain elongation by using di-isocyanates is always possible. Unfortunately, the ACE mechanism has some disadvantages e.g. a lack of molecular weight control and product reproducibility, poor initiator incorporation and formation of cyclic oligomers [36]. For these reasons, an Activated Monomer Mechanism (AMM), which involves the concept of living polymerization, where the OH-terminated polymer reacts with an "active" monomer, may be preferable since it avoids the formation of unstable and highly active cationic propagating species and favors the molecular weight control and reproducibility of the results. Side reactions, including cyclization, are strongly reduced in AMM and well-defined linear products can be obtained [7]. However, it must be emphasized that it is not easy to drive the reaction toward the desired mechanism and often both mechanisms may be present at the same time [37, 38].

It is important to introduce another aspect, related to safety during the synthetic process. In contrast to the above-mentioned polymers, the low molecular weight azide monomers are highly dangerous and may explode without apparent reasons during handling. In addition, the 
polymerization of energetic monomers requires careful control of reaction conditions since initiators may not be compatible with the energetic groups. Consequently, this polymerization is seen as an advanced technology and high-risk approach. Alternative to azidationpolymerization is the polymerization-azidation route, where the azidation step follows that of polymerization of the chlorinated-tosylated monomers. This is a low- tech., low-risk approach, but gives less opportunity to tailor the final properties of the polymer and suffers from the usual complications of modifying a macromolecule [36]. Taking into account the risks associated to the scale up for an industrial production, researchers are nowadays aiming toward the polymerization followed by azidation. With regard to the three candidate repeating units, BAMO is the one with the higher N content (50\%w) followed by GA (42 w \%) and AMMO $(33 \% \mathrm{w})$. However, BAMO is a symmetric monomer and gives a crystalline homopolymer that cannot be used as energetic binder, but is suitable to provide the hard block of an energetic thermoplastic elastomer [7, 39]. In contrast, GA and AMMO are nonsymmetric and provide amorphous character to the polymer. Therefore, the best solution to combine the amorphous character and the high energetic content seems to be the random copolymerization of BAMO with either GA or AMMO introduced in the minimum quantity necessary to break up the crystallization ability of the polymer. Barbieri et al described the synthesis of polyAMMO (PAMMO) and polyBAMO (PBAMO) homopolymers starting from the chlorinated and brominated precursors and followed by azidation with sodium azide in DMF [40]. In the same paper, poly(GA-r-BAMO) copolymers were also synthesized by using different molar ratios between the two repeating units. Later on, the GA/BAMO $=75 / 25$ molar ratio was considered as the optimal one and the effects of different operating conditions on copolymer characteristics were investigated [37]. Even though the operating conditions were set to favor a living character of the polymerization, the final product resulted in combined AMM and ACE mechanism. In particular, the latter is responsible for the formation 
of cyclic oligomers, which of course do not contribute to the formation of the binder network, but may be tolerated in the formulation since they behave as plasticizers perfectly miscible and compatible with the binder. The polymers were also subjected to preliminary characterizations in lab-scale propellant formulations [41, 42]. The same research group studied also the use of the tosylated precursor of AMMO to prepare PAMMO [43] and p(AMMO-r-BAMO) copolymers [38].

Pisharat et al. choose to produce a thermoplastic elastomer and prepared a poly(BAMO- $b$ GA- $b$-BAMO) block copolymer by using hydroxyl terminated $\mathrm{PECH}$ as initiator for the polymerization of the chlorinated precursor of BAMO. The azidation was then performed by using $\mathrm{NaN}_{3}$ in DMF [39].

Another possible strategy is the joining of HTPB and energetic binders, in order to keep the advantages of both components. However, as it is easily predictable, due to their different composition and polarity, HTPB and the azidic polyethers are not compatible and the mixture is destined to phase separation [44]. Nonetheless, Manu et al. prepared blends of HTPB and GAP and determined their glass transition after cross-linking with isocyanates [44]. The glass transition temperatures were evaluated through differential scanning calorimetry (DSC) and, even though the reported spectra are not easy to interpret, the authors claim that the blends show a "dominant single glass transition" that indicates a micro-heterogeneous morphology of the interpenetrating network. This result is somehow in agreement with those reported by Mathew et al. who did a mechanical and thermal characterization of cross-linked GAP/HTPB networks [45]. The glass transition temperatures were evaluated by dynamic mechanical analysis and it was found that blends prepared with GAP content up to $30 \%$ showed a single transition in the loss tangent trace. Very recently, Ding et al. used a triazole curing system as alternative to the traditional isocyanates, starting from GAP and a propargyl-terminated polybutadiene, under the catalysis of cuprous chloride at ambient temperature [46]. 
Nonetheless, it is not advisable to use HTPB and GAP or another azidic polymer in a simple blend that may separate before the curing process, thus giving the risk of inhomogeneity in the final product. A possible solution could be the use of a compatibilizer, which may be, e.g. a diblock copolymer made with the two components, but the best way to limit the problem of phase separation is the covalent bonding of the two polymers to form block copolymers. In block copolymers, usually the incompatibility between the blocks leads to a biphasic structure where the dispersed phase is organized in nanoscale blocks that may arrange in several configurations that have been widely studied both from the experimental and theoretical point of view [47]. Zhou et al. made a simulation study of the morphologies of GA- $b$-PB and GA- $b$ PB- $b$-GA di- and tri-block copolymers and concluded that there is a narrow range of compositions where a "bicontinuous" phase may form and improve the overall mechanical properties $[48,49]$. Since after copolymerization, the polybutadiene block does not contain the $\mathrm{OH}$ terminal groups, in the above sentence and in what follows it is indicated as PB, while HTPB refers to the unreacted $\mathrm{OH}$ terminated homopolymer.

The GAP/HTPB block copolymers have been synthesized and characterized by a few research groups. Eroğlu et al. first described the grafting of GAP onto HTPB, via free radical mechanism, by using a GAP "macroinitiator" obtained by reacting the azidic polymer with 4,4' azo bis(4-cyanopentanoyl chloride) [50]. Later on, Murali and Raju used the same procedure and made a detailed characterization of the graft copolymer, which exhibited two distinct glass transition temperatures at -74 and $-36{ }^{\circ} \mathrm{C}$, corresponding to the $\mathrm{PB}$ and GA blocks respectively [51].

Alternatively, the hydroxyl functionalities of HTPB may start the polymerization of a suitable monomer, thus directly giving a tri-block copolymer with PB as the central block. This was done for ECH almost contemporarily by Vasudevan and Sundararajan [52] and by Subramanian [53]. The main difference in the two procedures is the azidation step, done by 
using $\mathrm{NaN}_{3}$, in DMSO or in a mixture of dimethylacetamide and toluene. Similarly, Reddy et al. used HTPB as the central block and polymerized BAMO on it [54]. It is worth nothing that in this case the azidation followed by polymerization was chosen.

In this work, the same idea of using HTPB as the polyalcohol that starts the ionic polymerization was followed to prepare block copolymers to be subsequently azidated. As already pointed out, the azidation of ether precursors can be performed by using many different operating conditions and solvents. However, in this case we found that the unsaturated polybutadiene middle block may lead to an undesired cross-linking that impedes the subsequent use as binder. Therefore, it has been necessary to test different precursors, solvents and reaction temperatures to obtain appropriate operating conditions to solve this problem. A mixture of dimethylacetamide (DMAc) and toluene, as suggested by Subramanian [53], showed to be suitable to limit the cross-linking problems during azidation. Four block copolymers were then prepared by using the same procedure: 1) GAP-PB-GAP copolymer starting from a tosylated glycidol, 2) GA/BAMO-PB-GA/BAMO copolymer from ECH and 3,3-bis-(Brome methyl)oxetane (BBrMO, the brominated precursor of BAMO), 3) GA/BAMO-PB-GA/BAMO copolymer from tosylated glycidol (GT) and BBrMO and 4) AMMO-PB-AMMO copolymer starting from 3-tosyloxymethyl-3-methyl oxetane (TMMO). Therefore, polymers 2 and 3 are equal, with the difference just in the precursors. The first polymer (GAP-PB-GAP) was already synthesized by Vasudevan and Sundararajan [52] and by Subramanian [53], but starting from different precursors, while, to our knowledge, the other two polymers have not been described yet in the scientific literature. The synthetic pathways are described in Figure 1 (preparation of the monomers starting from the commercial products) and Figure 2 (synthesis of the polymeric precursors). In all cases, a polymeric non-energetic precursor was prepared and subsequently azidated. 
All the monomers and polymers were characterized by Fourier Transfer Infra Red (FTIR) analysis and Nuclear Magnetic Resonance (NMR). The thermal behavior of the polymers was evaluated by Differential Scanning Calorimetry (DSC) and Thermogravimetric Analysis (TGA).

\section{Materials and methods}

\subsection{Materials}

All the chemicals, unless differently stated, were purchased at Sigma Aldrich. N,N dimethylacetamide (99\%), toluene (99,8\%), dimethyl sulfoxide (DMSO) (99.7\%), dimethylformamide (DMF) (99.8\%), methanol (99.9\%), ethanol (99.9\%), sodium azide ( $>99.5 \%$ ), boron trifluoride tetrahydrofuranate (BTF THF), triethylamine (TEA) $(\geq 99 \%), 3-$ hydroxy-methyl-3-methyloxetane (HMMO) (98\%), glycidol (G) (96\%), toluene-4-sulfonyl chloride ( $\mathrm{TsCl}$ ) ( $\geq 99 \%$ ), sodium chloride ( $>99 \%$ ), sodium hydroxide ( $>98 \%$ ), sodium carbonate anhydrous, sodium bicarbonate (>99.7\%), magnesium sulfate $(>99.5 \%)$, phosphorus pentoxide, hydrochloric acid, potassium hydroxide were used as received. Diethyl-ether, 3-bromo-2,2-bis(bromomethyl)propanol (BrBBrMP) from Chemos GmbH, was used as received. Dichloromethane (DCM) was dried with $\mathrm{P}_{2} \mathrm{O}_{5}$ and distilled at $40^{\circ} \mathrm{C}$ and 1 atm, ECH and butanediol were distilled under reduced pressure. HTPB was Poly bd ${ }^{\circledR}$ R45HTLO by Cray Valley, with the following main properties: viscosity $=8000 \mathrm{mPa} . \mathrm{s}$ at 23 ${ }^{\circ} \mathrm{C}$, hydroxyl value $=0.84 \mathrm{meq} / \mathrm{g}$, hydroxyl functionality $=2.4-2.6 \mathrm{OH}$ groups $/ \mathrm{chain}$, glass transition temperature $=-75^{\circ} \mathrm{C} .2,2^{\prime}$-Methylenebis(6-tert-butyl-4-methylphenol) used as antioxidant was VulKanox BKF by Lanxess. 


\subsection{Synthesis of monomers}

\subsubsection{Glycidyl tosylate GT}

The synthesis was performed according to a procedure described by Nakabayashi et al [55]. A $250 \mathrm{~mL}$ flask, was fed with $50 \mathrm{~mL}$ of anhydrous toluene and $4.2 \mathrm{~mL}$ of TEA. In a beaker, a solution of $5.42 \mathrm{~g} \mathrm{TsCl}$ in $12 \mathrm{~mL}$ of toluene was also prepared. The flask and the beaker were then conditioned at $-15^{\circ} \mathrm{C}$ for $1.5 \mathrm{~h}$. Then, the flask was fed with $2 \mathrm{~mL}$ of distilled $\mathrm{G}$ and dropwise with the $\mathrm{TsCl}$ solution. At the end of the feeding, the system was maintained at $-15^{\circ} \mathrm{C}$ for $24 \mathrm{~h}$, filtered and distilled. The remaining liquid was dropped in cold petroleum-ether thus forming a white suspension that crystallizes at $-15^{\circ} \mathrm{C}$. The GT was obtained with a $98.6 \%$ yield, as white anhydrous crystals.

\subsubsection{3,3-bis(bromomethyl)oxetane (BBrMO)}

A $1 \mathrm{~L}$ three-necked round bottom flask, fitted with a reflux condenser, a nitrogen inlet and a mechanical stirrer set at $180 \mathrm{rpm}$ was fed with $50 \mathrm{~g}$ di BrBBrMP and $100 \mathrm{~mL}$ of ethanol. The flask was then immersed in a water bath set at $6 \pm 1^{\circ} \mathrm{C}$ and $100 \mathrm{~mL}$ of a $1.77 \mathrm{M}$ solution of $\mathrm{NaOH}$ in ethanol were added drop by drop in about $0.5 \mathrm{~h}$. Once all the $\mathrm{NaOH}$ solution was fed to the reactor, the bath temperature raised to $70{ }^{\circ} \mathrm{C}$ and the solution was kept under stirring for further $1 \mathrm{~h}$. Then, the solution was cooled to room temperature, filtered under reduced pressure $(6500 \mathrm{~Pa})$ and mixed with $50 \mathrm{~mL}$ of DCM. The organic phase was washed several times with distilled water and BBrMO was finally obtained after distillation at 30,000 Pa and $61{ }^{\circ} \mathrm{C}$, with a $93.0 \%$ yield.

\subsubsection{3-tosyloxymethyl-3-methyl oxetane (TMMO)}

The synthesis was performed in the solid state, according to a procedure described by Kazemi et al [56]. A mortar was fed with $31.8 \mathrm{~g}$ of anhydrous $\mathrm{Na}_{2} \mathrm{CO}_{3}, 10.2 \mathrm{~g}$ of $\mathrm{HMMO}$ 
$\left(\mathrm{Na}_{2} \mathrm{CO}_{3} / \mathrm{HMMO}=3 / 1\right.$ molar ratio $)$ and manually milled with a pestle for about $7 \mathrm{~min}$. Then $28.7 \mathrm{~g}$ of $\mathrm{TsCl}(\mathrm{TsCl} / \mathrm{HMMO}=1.5 / 1$ molar ratio) where added and milled for another $23 \mathrm{~min}$. Finally, $28.05 \mathrm{~g}$ of $\mathrm{KOH}$ were added $(\mathrm{KOH} / \mathrm{HMMO}=5 / 1$ molar ratio) and milled for about 50 min to remove the unreacted $\mathrm{TsCl}$. The whole reaction was conducted in a glove box under nitrogen atmosphere. The obtained solid was added to $200 \mathrm{~mL}$ of diethyl-ether and filtered several times. The obtained clear liquid phase was finally subjected to distillation at $48^{\circ} \mathrm{C}$ and $8500 \mathrm{~Pa}$ thus inducing the crystallization of TMMO that was obtained with a $40 \%$ yield.

The structure and purity of all monomers was verified by Fourier transform infrared spectroscopy (FTIR) and by nuclear magnetic resonance $\left({ }^{1} \mathrm{H}-\mathrm{NMR}\right)$.

\subsection{Polymerizations}

As stated above, the block copolymers formed through the growing of the energetic segments on each end of preformed HTPB, act as initiator. The catalyst and operating conditions were set in order to favor the cationic ring opening polymerization by AMM. In all the polymerizations, the quantities of BTF THF and HTPB were such that the molar ratio between BTF THF and the $\mathrm{OH}$ functionalities of the polymer was equal to $2 / 1$, while the molar ratio between monomers and $\mathrm{OH}$ functionalities was equal to 50/1. Table 1 reports the quantities used for a typical polymerization of each block copolymers, being in the last column indicated the theoretical $\mathrm{N}_{3}$ content after azidation, calculated from the hypothesis of $100 \%$ yield in both the polymerization and azidation reactions. The polymerization procedure was as follows: A 1L three-necked round bottom flask, fitted with a reflux condenser, a nitrogen inlet and a mechanical stirrer set at $120 \mathrm{rpm}$ was fed with $80 \mathrm{ml}$ of DCM, $0.72 \mathrm{~mL}$ of BTF THF, about $4 \mathrm{~g}$ of HTPB and maintained $2 \mathrm{~h}$ under stirring at room temperature. Then, the reactor was covered with aluminum foil, immersed in a water bath at $20 \pm 0.5^{\circ} \mathrm{C}$ and the 
monomers, previously dissolved in $30 \mathrm{~mL}$ of DCM, were added drop-wise to the reactor through a dropping funnel. From the end of the monomer feeding, the reaction mixture was kept in the same conditions for $20 \mathrm{~h}$ (120 h when using tosylated monomers) and then hydrolyzed with $400 \mathrm{~mL}$ of an aqueous solution of $\mathrm{NaCl}(10 \% \mathrm{w})$. The mixture was maintained under vigorous stirring for further $2 \mathrm{~h}$ and then the organic and aqueous phases were separated. The aqueous phase was washed with DCM, subsequently recovered and added to the organic phase. The organic phase was washed once with a water/methanol= $50 / 50 \mathrm{v} / \mathrm{v}$ solution to remove unreacted monomers and catalyst, then several times with the aqueous solution of $\mathrm{NaCl}$, dried with $\mathrm{MgSO}_{4}$, filtered and distilled at $45^{\circ} \mathrm{C}$, under vacuum (6500 Pa) in order to remove all the DCM. All the polymers were obtained with a 95-97\% yield.

\subsection{Azidation of the polymeric precursors}

The azidation was conducted with the well-known second-order reaction with SN2 type mechanism, by using sodium azide and a polar solvent. This technique of azidation is almost "universal" and in the last decades many researchers, which tested several solvents and operating conditions as well as many different leaving groups, described it. In our research group, it was previously used to substitute chlorine, bromine and tosyl groups, in either DMF or DMSO, with temperatures ranging from 90 up to $150^{\circ} \mathrm{C}[37,38,40,43]$. Pisharat and Ang azidated PECH in DMF, at $120^{\circ} \mathrm{C}$ for $12 \mathrm{~h}$ [39], while Manser suggested DMSO at $80{ }^{\circ} \mathrm{C}$ also for the azidation of the oxetanic monomers [33]. Many other examples of azidic compounds can be found in the literature, with DMF and DMSO the most used solvents. However, we found that neither DMF nor DMSO were suitable for the azidation step, because before obtaining the complete azidation of the polymers, an incipient cross-linking reaction was observed, thus leading to partially soluble materials. This happened in several attempts made 
at testing temperatures ranging from 100 to $130^{\circ} \mathrm{C}$. The addition of small quantities of Vulkanox BKF as antioxidant only reduced the phenomenon. Since this procedure is well established and was already tested several times in our laboratory for GAP, BAMO and PAMMO polymers and copolymers, which never caused such inconvenience, the crosslinking must be correlated with the presence of the unsaturations in the polybutadiene middle block. However, Vasudevan and Sundararajan, which used DMSO at $105^{\circ} \mathrm{C}$ for $10 \mathrm{~h}$, did not describe the cross-linking problem in their work [52]. In our case, the use of a toluene/DMAc mixture, as suggested by Subramanian [53], in combination with small quantities of Vulkanox BKF, gave good results and was adopted for all azidations.

A typical azidation procedure was as follows: About $2 \mathrm{~g}$ of polymeric precursor were dissolved in $100 \mathrm{ml}$ of DMAc/toluene $(50 / 50 \mathrm{v} / \mathrm{v})$ solution and fed in a $250 \mathrm{~mL}$ three-necked round bottom flask, fitted with a reflux condenser, a nitrogen inlet and a magnetic stirrer. After adding a small quantity of Vulkanox BKF (about $1 \%$ by weight with respect to the polymer), the flask was immersed in an oil bath set at $90 \pm 1^{\circ} \mathrm{C}$ and conditioned for 30 minutes. Then, $\mathrm{NaN}_{3}$ was added in $10 \%$ molar excess with respect to the stoichiometric quantity and the temperature of the bath raised to $95^{\circ} \mathrm{C}$ while maintaining the system under constant stirring. Periodically, samples were taken from the reactor and subjected to FTIR analysis to evaluate the degree of azidation. Once the IR spectra reached a stationary "state", the reaction medium was filtered under vacuum in order to remove the formed sodium salt's and the unreacted sodium azide. The reaction time strongly varied depending on the monomeric precursors (Table 2).

The solution was then washed several times with an aqueous solution of $\mathrm{NaCl}(10 \% \mathrm{w})$ in order to complete the salts removal. The aqueous phases resulting from the washings were mixed together and washed with DCM to remove possible traces of polymer, while the organic phase was dried with $\mathrm{MgSO}_{4}$, filtered again and distilled at $45^{\circ} \mathrm{C}$, under vacuum 
(1000 Pa) in order to remove all the organic solvents. A small quantity (about $1.5 \%$ by weight) of Vulkanox BKF was finally added to the polymer before storage. All the azidated copolymers were obtained with a yield higher than $95 \%$.

The structure and purity of all synthesized polymers (before and after azidation) was checked by FTIR and NMR, while their thermal properties were determined by DSC and TGA.

\subsection{Characterization of the monomers and polymers}

Fourier transform infrared spectroscopy was performed on a Bruker Tensor 27 and nuclear magnetic resonance $\left({ }^{1} \mathrm{H}-\mathrm{NMR},{ }^{13} \mathrm{C}-\mathrm{NMR}\right)$ on a VXR300 and INOVA600 instruments.

Chemical analysis and FTIR Chemical Imaging were carried out by Spectrum Spotlight FTIR Imaging System from Perkin Elmer. Chemical imaging analysis, in transmittance mode, allowed combining optical microscopy and infrared analysis of micro and macro areas of thin films prepared by solvent evaporation on a microscope slide. The morphological analysis was performed also by a LEICA DM LB fluorescence microscope. The molecular weight distributions of the polymers were measured from solution in $\mathrm{CHCl}_{3}(4 \mathrm{mg} / \mathrm{mL})$ by using a Gel Permeation Chromatography (GPC) apparatus Jasco PU-1580, equipped with PL Mesopore column, calibrated with low polydispersity polystyrene standards. Thermogravimetric analysis (TGA) was done by using a TA Q500 apparatus, under nitrogen atmosphere, with a heating rate of $10^{\circ} \mathrm{C} / \mathrm{min}$ until $600{ }^{\circ} \mathrm{C}$ and using samples of about $5 \mathrm{mg}$. Thermal properties were studied by differential scanning calorimetry (DSC) performed with a Pyris 1 scanning calorimeter from Perkin Elmer, by using aluminum pans. The sample mass was in the range of $3-5 \mathrm{mg}$ and spectra were collected from $50{ }^{\circ} \mathrm{C}$ at a heating rate of $10^{\circ} \mathrm{C} / \mathrm{min}$, until $350^{\circ} \mathrm{C}$.

\section{Results and discussion}




\subsection{Chemical characterization (IR - NMR)}

IR and NMR spectra were recorded for all monomers, precursors and polymers synthesized in this work. In order to limit the number of figures, we report only two H-NMR spectra, showing the comparison between GT/BBrMO-PB-GT/BBrMO and GA/BAMO-PBGA/BAMO in the range corresponding to the tosyl group signals (Figure 3).

With regard to the IR spectra, they are reported in Figures 4 and 5, showing the main peaks involved in the azidation process. In all cases, but one, azidation appeared to be quantitative. The only polymer without complete substitution of the leaving groups is AMMO-PB-AMMO, where the peaks relative to the tosyl group are clearly visible even after $140 \mathrm{~h}$ of azidation reaction. A quantitative evaluation of the residual tosyl groups indicates a degree of azidation close to $50 \%$. The peaks highlighted in the figures are: $554 \mathrm{~cm}^{-1}=\mathrm{N}_{3}$ bending, $670 \mathrm{~cm}^{-1}=\mathrm{C}$ Br stretching, $744 \mathrm{~cm}^{-1}=\mathrm{C}-\mathrm{Cl}$ stretching, $1190 \mathrm{~cm}^{-1}=\mathrm{SO}_{2}$ symmetric stretching, $1280 \mathrm{~cm}^{-1}=$ $\mathrm{N}_{3}$ symmetric stretching, $1363 \mathrm{~cm}^{-1}=\mathrm{SO}_{2}$ asymmetric stretching, $1600 \mathrm{~cm}^{-1}=$ aromatic $\mathrm{C}=\mathrm{C}$ vibration, $2100 \mathrm{~cm}^{-1}=\mathrm{N}_{3}$ asymmetric stretching.

From the reaction times reported in Table 2, it can be concluded that the azidation of the tosyl group is the bottleneck of the reported synthetic strategies. This is somehow unexpected, because the tosyl is a good leaving group. However, it should be considered that the group is attached to a polymeric chain, so there are several aromatic rings close one to each other and this may determine a high steric hindrance as well as a low mobility of the chains even when in solution. In the case of the GT/BBrMO copolymer, probably the alternation of GT and BBrMO groups allows sufficient mobility of the chain, so that a complete azidation was obtained in a short time. Therefore, even if potentially better than halogen atoms, the tosyl group may determine a slowing down of the azidation kinetics and also limits the maximum degree of azidation obtainable within the reaction times adopted in this work. 
An interesting point concerning FTIR is that a comparison of the GA-PB-GA spectrum (labeled as N. 6 in Figure 5) with the corresponding one reported in the paper by Subramanian [53] clearly shows that in the latter the $\mathrm{N}_{3}$ peak is significantly smaller. This means that the synthetic procedure adopted in this work enables formation of copolymers with a significantly higher azide content.

With regard to the characterization of the copolymers, during the revision process it was asked if the alcohol moieties in the HTPB chain were really used as the primary initiator site rather than adventitious moisture. In the latter case, we would have obtained the polyether instead of a block copolymer. Therefore, depending on the primary initiator, the final product may contain block copolymer, polyether and HTPB. Block copolymer alone is the desired product, with all HTPB used as initiator and no moisture involved. A mechanical mixture of block copolymer and polyether will form if both HTPB and moisture act as initiator. A mechanical mixture of block copolymer and HTPB is obtained if HTPB is only partially used as initiator. A mechanical mixture of HTPB and polyether is the worst case, with no HTPB used as initiator. And so on, with other combinations possible. To clarify this point, we can start from the above-described incompatibility between the ether and butadiene blocks [44], which leads to a macroscopic phase separation when blended. For this reason, the morphologies of the polymeric precursors were compared with those of the mechanical blends prepared from the polymers constituting the single blocks. For this purpose, the ether blocks were appositely synthesized, by using the same synthetic strategy described for the block copolymers, simply substituting HTPB with butanediol. Particular care was taken in order to assure the use of identical operating conditions during polymerizations (reagent purity, volumes, solvent quantities and so on). The mechanical blends (MB) were prepared by solvent evaporation from a DCM solution where HTPB and polyether were added in quantities corresponding to the composition calculated from the yields of the copolymer 
synthesis. Moreover, MB and copolymers were subjected to fluorescence microscopy and Spectrum Spotlight FTIR Imaging. As an example of the obtained results (qualitatively similar for all the synthesis and corresponding MB), Figure 6 shows the images and spectra recorded for PGT and HTPB. The MB is on the left hand side of the Figure and the product of the synthesis (named product in what follows) is on the right hand side. The fluorescence microscopies of MB (Figure 6a) and the product (Figure 6b) are completely different. The latter is quite homogeneous and shows some area with slightly different fluorescence intensity, but no visible phase separation. In contrast, the MB is markedly biphasic, with dark roundish drops dispersed in a bright matrix. A comparison of these images with those (not reported) of the homopolymers suggests that the dark phase corresponds to HTPB. However, the dark phase and brightness inhomogeneity may also derive from voids and/or variations of the sample thickness. Therefore, the samples were analyzed by Spectrum Spotlight FTIR Imaging, which uses optical microscopy (Figures $6 \mathrm{c}$ and $6 \mathrm{~d}$ ) and allows building a chemical maps (Figures 6e and 6f). The maps represent the normalized peak area between 2980 and $2700 \mathrm{~cm}^{-1}$ (stretching of aliphatic C-H bonds). This peak is present in both polyether and HTPB, but its intensity is significantly higher in the latter. Therefore, the grey level in the map reflects the composition of the sample: a high HTPB content corresponds to white color in the map. Figure 6e shows areas with markedly different grey level and confirms that the round domains of MB are mainly composed of HTPB. In contrast, Figure $6 \mathrm{f}$ does not show any significant variations in grey level. Moreover, the arrows indicate the spectra recorded in correspondence of the "十" symbols reported in Figures 6e and 6f. The two spectra on the left hand side differ one from each other and result similar to those of the polyether and HTPB homopolymers (not reported). In contrast, the right hand side spectra are very similar, even if recorded in areas corresponding to the maximum possible difference in grey scale. Based on the IR spectra and maps, we can conclude that the shadows and apparent inhomogeneity 
observable in Figures $6 \mathrm{~b}, 6 \mathrm{~d}$ and $6 \mathrm{f}$ do not correspond to significant variation in chemical composition. Therefore, we can exclude that the reaction product is a mechanical mixture of HTPB and polyether.

Moreover, a phase separation is observable also while adding very small quantities of HTPB to the product. This excludes also that the product could be a mechanical mixture of block copolymer and HTPB. The last proof that all HTPB was used to build the polyether blocks comes from an extraction with n-hexane. The mechanical blend was immersed in n-hexane, and stirred vigorously at room temperature for a few minutes, after which a consistent part of the blend showed to be immiscible with the solvent. Then, the hexane-phase was recovered and the solvent evaporated. The resulting polymeric phase was HTPB alone. The same procedure was followed for the copolymer and, in that case, no HTPB was recovered after hexane evaporation.

The presented results demonstrate that HTPB worked as initiator for the polyether, but leave the doubt that the ether could polymerize starting from the above-mentioned water moieties. This possibility is validated from the fact that the addition of small quantities of polyether to the synthesis product did not give phase separation (contrary to what happened while adding HTPB). Unfortunately, this hypothesis could not be verified with solvent extraction, because we did not find a solvent selective toward the ether phase. Several polar solvents were tried, but all of them partially dissolved the HTPB, probably due to its $\mathrm{OH}$ functionalities. A more enhanced extraction procedure was necessary and the GPC column showed to be useful. As an example, Figure 7 shows the GPC analysis of HTPB and GT-PB-GT copolymer. The weight molecular weight distribution of the copolymer resulted bimodal, and the curve was subjected to deconvolution (dashed lines). The obtained results are summarized in Table 3 and show that the right hand peak of the copolymer has a molecular weight slightly higher than HTPB. In contrast, the left hand peak has a very low molecular weight, thus 
remembering the values usually associated with the formation of cyclic oligomers composed of 3-5 repeating units [40]. Therefore, the correct interpretation of the GPC is that the highmolecular weight peak is the block copolymer, while the low-molecular weight peak is due to the presence of polyether not linked to HTPB and grown from $\mathrm{OH}$ functionalities of different origin. The synthesis product is a mechanical mixture of block copolymer and polyether, thus both HTPB and some adventitious moisture worked as initiator. The polyether content was calculated from the area of the peaks and the results are reported in the last column of Table 3. In all cases, the oligomer content is around $20 \%$ by weight of the total amount.

We can thus affirm that all the HTPB chains acted as initiator for the polymerization, but some of the $\mathrm{OH}$ moieties present in the reaction medium did the same and led to the formation of polyether chains not covalently linked to the polybutadiene. The product thus contains polyether oligomers that are difficult to separate from the copolymer. Nevertheless, if these oligomers are linear, they should be $\mathrm{OH}$ functionalized and participate to the curing with isocyanates while forming the polymeric network that constitutes the final binder. Otherwise, in case they are cyclic oligomer, they will act as plasticizers in the binder formulation.

\subsection{Thermal characterization (DSC - TGA)}

In the scientific literature there is a long list of papers that analyze the thermal decomposition of polymers (especially GAP) containing the azidic functionalities, alone or after curing and addition of plasticizers $[17,20,21,23,25,31,57]$. Usually, the easiest way to determine the thermal stability of this kind of polymers is by TGA and DSC analysis. However, due to the violent kinetics of decomposition, it may not be easy to obtain reliable and reproducible results. Selim et al., in the case of GAP, used 2-3-mg samples, and kept the ramp rates in the 
range of $0.2-5^{\circ} \mathrm{C} / \mathrm{min}$ since a greater amount of polymer or higher heating rate lead to explosion during the decomposition experiment [14]. In all cases, while subjected to a temperature sweep procedure, the azidic polymers degrade in a two-stage process. The first one, occurring at about $240-270{ }^{\circ} \mathrm{C}$, is exothermic and corresponds to the abstraction of $\mathrm{N}_{3}$ groups from the polymeric chain. The second one, partially overlapped with the first one, but shifted to higher temperatures, generally occurs without significant heat release and corresponds to the progressive decomposition of the remaining polymeric chain. In one of the first and most cited work, Kubota and Sonobe suggested that the first process corresponds to the release of a nitrogen molecule from the $\mathrm{N}_{3}$ group, with the formation of a nitrile $\mathrm{C} \equiv \mathrm{N}$ bond [58]. The following studies confirmed that the decomposition starts with the initial rupture of $\mathrm{N}-\mathrm{N}_{2}$ bond, with elimination of molecular nitrogen. Then, rearrangement to a polymeric imine and/or acrylonitrile following elimination of hydrogen molecule has been proposed. In the final products of decomposition, gaseous $\left(\mathrm{N}_{2}, \mathrm{CO}, \mathrm{HCN}, \mathrm{NH}_{3}, \mathrm{CH}_{2} \mathrm{O}, \mathrm{CH}_{4}\right.$, $\mathrm{C}_{2} \mathrm{H}_{2}, \mathrm{C}_{2} \mathrm{H}_{4}, \mathrm{CH}_{3} \mathrm{CHO}, \mathrm{CH}_{2} \mathrm{CHCHNH}, \mathrm{CH}_{3} \mathrm{CHNH}, \mathrm{H}_{2} \mathrm{O}$ etc.) and larger molecules as benzene, pyrrole and furan have been identified [18, 23, 24]. However, in all cases, the weight loss experimentally measured during the first stage is higher than the theoretical one based on sole $\mathrm{N}_{2}$ release. This is due to the above-mentioned partial overlapping with the incipient second stage of decomposition, but also to the occurrence of other, parallel reactions during the first stage. Various mechanisms and possible involved reactions have been proposed by analyzing the residual solid and the evolved gases during decomposition. As an example, Eroĝlu and Güven used FTIR and observed that the decrease of $\mathrm{N}_{3}$ content was accompanied by the appearance of signals related to $\mathrm{N}-\mathrm{H}$ bending, $-\mathrm{C}=\mathrm{N}-\mathrm{C}-\mathrm{H},-\mathrm{C}=\mathrm{N}-\mathrm{C}-\mathrm{C}$, and $\mathrm{N}-\mathrm{H}$ stretching. At the same time, the signal of the $\mathrm{C}-\mathrm{O}-\mathrm{C}$ ether bridge remained unchanged thus suggesting that the main chain was not yet involved in the decomposition process. Based on these observations, a mechanism based on the formation of intra and inter-molecular cross- 
linking was suggested for the first stage of degradation [13]. Reshmi et al. used DSC and TGA coupled with pyrolysis gas chromatography-mass spectrometric technique and showed that during its first stage of decomposition, 1,6-bis (azidoacetoyloxy) hexane preferentially forms the corresponding diimine by elimination of $\mathrm{N}_{2}$ [59]. Then, at higher temperatures, there is formation of diimines by elimination of $\mathrm{CO}_{2}$, diols through elimination of $\mathrm{CO}$ and $\mathrm{HCN}$ and diene due to $\mathrm{CO}_{2}$ and $\mathrm{CH}_{2} \mathrm{NH}$ elimination. The photodegradation under ultraviolet (UV) irradiation was also investigated by Sahu et al. [15] and by Wang et al. [19]. The latter combined a tunable synchrotron vacuum ultraviolet photoionization and molecular-beam sampling mass spectrometry and observed that the UV radiation lowered the onset of the thermal decomposition of GAP and caused the appearance of a larger number of free radical species, compared to thermal decomposition alone.

The DSC and TGA analysis are reported in Figures 8-10 and summarized in Tables 4 and 5. In both Tables, $T_{i}$ and $T_{f}$ indicate the initial and final temperatures of the degradation step. The produced heat and weight loss are reported after normalization with respect to both the total weight and the theoretical $\mathrm{N}_{3}$ content of the polymer. In Table $5, \Delta \mathrm{w}$ is the weight loss with respect to the total initial mass and $\Delta \mathrm{w} \mathrm{N}_{3}(\%)$ is the weight loss compared to the theoretical one expected if all azidic groups release one $\mathrm{N}_{2}$ molecule. Finally, $\mathrm{W}_{\mathrm{r}}$ is the residual weight at the end of the test $\left(600^{\circ} \mathrm{C}\right)$. The DSC traces are reported only for the azidic polymers. It is useful to underline that such high temperatures have only scientific interest, not practical, because the behavior of the polymers in propellant will not be the same as in the individual state due to the oxidizer presence around.

As already stated, the $\mathrm{N}_{3}$ group decomposition is responsible for the exothermic peak observed in DSC and the first weight loss in the TGA thermograms. However, this decomposition may follow different pathways and be partially superposed to other degradation phenomena, which depend not only on the operating conditions, but also on the 
polymer structure. Therefore, the weight loss and energy release during this stage are unequivocally determined in the literature. As an example, in the case of GAP, the following values (here expressed as $\mathrm{J} / \mathrm{g}$ of $\mathrm{N}_{3}$ ) were reported: 4,352 [13], 4,835 [14] and 6,166 [21], representing the heat of formation of the azido group around $8500 \mathrm{~J} / \mathrm{g}$ of $\mathrm{N}_{3}$ [27]. In our case, the decomposition is probably "complicated" by the presence of the butadiene block that may interact with the formed species. The enthalpies reported in Table 4 are substantially in agreement with those reported in the literature. The value obtained for the AMMO-PBAMMO copolymer confirms the estimation of about $50 \%$ substituted tosyl groups. Moreover, in the spectrum of this polymer there is the superposition with a second peak (centered at about $275{ }^{\circ} \mathrm{C}$ ) that corresponds to the exothermic degradation of the tosyl group and well visible in the spectra of the tosylated precursor (not reported here).

With regard to TGA (Figures 9 and 10), a first general observation is that all the azidated copolymers, but AMMO-PB-AMMO, have a significant residual weight at $600{ }^{\circ} \mathrm{C}$ (see last column of Table 5). In contrast, the precursors show a much lower final weight and HTPB alone has almost no residual weight at the same final temperature. This difference between azidated polymers and precursors suggests that during thermal decomposition some chemical interactions may occur between the azidic functionalities and the butadiene backbone.

Probably, this is strictly connected with the above-mentioned tendency of the copolymers to cross-link during azidation and subsequent storage. It may be supposed that the first decomposition products interact with the butadiene main chain and form a highly cross-linked network that slows down the degradation process at higher temperatures. Of course, the explanation of different behavior of the AMMO-PB-AMMO copolymer can be based on its low degree of azidation. This difference among azidated copolymer and non-energetic precursors is coherent with the shape of the TGA curves. The precursors show a two-step degradation, well separated by a horizontal plateau. A comparison with the HTPB curve 
suggests that the first step is relative to the ether block, while the second one is the butadiene block. The azidic copolymers show again a two-step degradation, but differently shaped. The first step, already attributed to the $\mathrm{N}_{3}$ functionality, is sharp, while the second one corresponds to a gradual weight loss that extends in a wide temperature range and ends at about $500{ }^{\circ} \mathrm{C}$. Again, AMMO-PB-AMMO copolymer is the only one with an intermediate behavior (curve 8 in Figure 10 shows three distinct steps).

The weight losses during the first decomposition step are higher than expected if each $\mathrm{N}_{3}$ functionality releases one nitrogen molecule (Table 5). This confirms that the main chain is already subjected to other degradation phenomena (mainly ascribable to the ether block) that superpose to the degradation of the azidic functionalities.

A last point to be underlined is that polymers 2 and 3 in Figure 9 have very slight differences in the TGA and DSC curves, which is a further confirmation of their substantial equivalence. The AMMO-PB-AMMO copolymer is the one that shows the lower enthalpy release during decomposition of the azidic functionalities. This is in accordance with the already observed incomplete substitution of the tosyl functionalities with the azidic ones. From DSC and FTIR we already calculated that this corresponds to about one-half of the theoretical azidic functionalities. However, this value must be analyzed by taking into account also the TGA curves. The TMMO-PB-TMMO precursor shows two very distinct and sharp peaks corresponding to the degradations of the ether and butadiene blocks. However, the weight loss $(16.6 \%)$ associated to the latter is higher than the theoretical value $(8.0 \%)$ corresponding to a $100 \%$ conversion during polymerization, thus suggesting that the TMMO blocks may be shorter than expected. Therefore, with respect to the expected structure, the AMMO-PBAMMO copolymer may have both shorter ether blocks and incomplete degree of tosyl functionalities. After $\mathrm{N}_{3}$ decomposition, the weight loss of the azidic copolymer shows two partially superposed consecutive steps related to ether and HTPB blocks degradation. 


\section{Conclusions}

The synthesis of ether-butadiene-ether energetic block copolymers was done by a classical cationic homo and co-polymerization of oxetanes and oxiranes, using HTPB as diol-initiator and followed by the azidation process. Four copolymers were synthesized, with the external linear blocks constituted by azido homo and copolymers. Probably due to the presence of water moieties, a small amount of polyether oligomers not linked with HTPB were also obtained as undesired side products. With respect to the corresponding polymers prepared by using a low molecular weight, saturated diol, such as i.e. 1-4 butanediol, the use of HTPB complicates the synthetic procedure, since the azide could have a side reaction with the double bonds to cause some species, which could lead to cross-linking. This may lead to the formation of a cross-linked block copolymer, which, of course, is not suitable as polymeric binder for solid propellants. The cross-linking phenomenon may happen either during the polymerization, azidation or purification steps, or also during prolonged storage at room temperature. This restricts the range of operating conditions available for the synthetic process, limiting the suitable solvents, as well as the temperatures and duration of the reactions and purification procedures. Moreover, the addition of an anti-ageing compound to the final product and the storage in an inert and dark atmosphere is necessary. The tosyl group may slow down the kinetics of the azidation step and may also lead to incomplete substitution. In conclusion, the synthesis of these azidic copolymers with an internal unsaturated block is not simple and presents several drawbacks not easy to manage. Nevertheless, the appropriate choice of the operating conditions allows these drawbacks to be partially overcome and results in a versatile tool for the synthesis of polymeric binders, whose properties can be modulated by changing the type and length of the ether blocks. 


\section{References}

[1] Colclough, M. E., H. Desai, R. W. Millar, N. C. Paul, M. J. Stewart, and P. Golding. 1993. Energetic polymers as binders in composite propellants and explosives. Polymers for Advanced Technologies, 5:554-560.

[2] Provatas, A. 2000. Energetic polymers and plasticisers for explosive formulations - A review of recent advances, DSTO-TR-0966.

[3] Sikder, A. K., and S. Reddy. 2013. Review on energetic thermoplastic elastomers (ETPEs) for military science. Propellants Explosives and Pyrotechnics, 38:14-28.

[4] Agrawal, J. P. 1998. Recent trends in high-energy materials. Progress in energy and combustion science, 24:1-30.

[5] Badgujar, D. M., M. B. Talawar, S. N. Asthana, and P. P. Mahulikar. 2008. Advances in science and technology of modern energetic materials: An overview. Journal of Hazardous Materials, 151:289-305.

[6] Sikder, A. K., and N. Sikder. 2004. A review of advanced high performance, insensitive and thermally stable energetic materials emerging for military and space applications. Journal of Hazardous Materials, A112:1-15.

[7] Gaur, B., B. Lochab, V. Choudhary, and I. K. Varma. 2003. Azido polymers-energetic binders for solid rocket propellants. Journal of Macromolecular Science, Part CPolymer Reviews, C43(4):505- 545.

[8] Chien, J. C. W., T. Kohara, C. P. Lillya, T. Sarubbi, B-H. Su, and R. S. Miller. 1980. Phase transfer-catalyzed nitromercuration of diene polymers. J. Polym. Sci. Part A Polym. Chem., 18(8):2723-2729. 
[9] Korobeinichev, O.P., L.V.Kuibida, E. N. Volkov, and A. G. Shmakov. 2002. Mass spectrometric study of combustion and thermal decomposition of GAP. Combustion and Flame, 129(1-2):136-150.

[10] Sahu, S.K., S. P. Panda, D. S. Sadafule, C. G. Kumbhar, S. G. Kulkarni, and J. V. Thakur. 1998. Thermal and photodegradation of glycidyl azide polymers. Polymer Degradation and Stability, 62:495-500.

[11] You, J. S., and S. T. Noh. 2013. Rheological and thermal properties of glycidyl azide polyol-based energetic thermoplastic polyurethane elastomers. Polym. Int., 62:158164.

[12] Manu, S. K., V. Sekkar, K. J. Scariah, T. L. Varghese, and S. J. Mathew. 2008. Kinetics of glycidyl azide polymer-based urethane network formation. Appl. Polym. Sci110:908-914.

[13] Eroĝlu, M. S., and O. Güven. 1996. Thermal decomposition of poly(glycidyl azide) as studied by high-temperature FTIR and thermogravimetry. Journal of Applied Polymer Science, 61:201-206.

[14] Selim, K., S. ÖZkar, and L. Yilmaz. 2000. Thermal characterization of glycidyl azide polymer (GAP) and GAP-based binders for composite propellants. Journal of Applied Polymer Science, 77:538-546.

[15] Sahu, S. K., S. P. Panda, D. S. Sadafule, C. G. Kumbhar, S. G. Kulkarni, and J. V. Thakur. 1998. Thermal and photodegradation of glycidyl azide polymers. Polymer Degradation and Stability, 62:495-500.

[16] Menke, K., J. Bohnlein-Mauß, and H. Schubert. 1996. Characteristic properties of AN / GAP-Propellants. Propellants, Explosives, Pyrotechnics, 21:139-145.

[17] Sun, Y., and S. Li. 2008. The effect of nitrate esters on the thermal decomposition mechanism of GAP. Journal of Hazardous Materials, 154:112-117. 
[18] Fazlioğlu, H., and J. Hacaloğlu. 2002. Thermal decomposition of glycidyl azide polymer by direct insertion probe mass spectrometry. Journal of Analytical and Applied Pyrolysis, 63:327-338.

[19] Wang, T., S. Li, B. Yang, C. Huang, and Y. J. Li. 2007. Thermal decomposition of glycidyl azide polymer studied by synchrotron photoionization mass spectrometry. Phys. Chem. B, 111:2449-2455.

[20] Arisawa, H., and T. B. Brill. 1998. Thermal decomposition of energetic materials 71: Structure- decomposition and kinetic relationships in flash pyrolysis of gliycidyl azide polymer (GAP). Combustion and Flame, 112:533-544.

[21] Feng, H. T., K. J. Mintz, R. A. Augsten, and D. E. G. Jones. 1998. Thermal analysis of branched GAP. Thermochimica Acta, 311:105-111.

[22] Tamura G., M. Tanabe, and T. Kuwahara. 2012. Decomposition of GAP single droplets used as liquid monopropellants. Propellants Explosives and Pyrotechnics, 37:302-307.

[23] Tang, C. J., Y. Lee, and T. A. Litzinger. 1999. Simultaneous temperature and species measurements of the glycidyl azide polymer (GAP) propellant during laser-induced decomposition. Combustion and Flame, 117:244-256.

[24] Korobeinichev, O. P., L. V. Kuibida, E. N. Volkov, and A. G. Shmakov. 2002. Mass spectrometric study of combustion and thermal decomposition of GAP. Combustion and Flame, 129:136-150.

[25] Laviolette, M., and M. Auger. 1999. Monitoring the aging dynamics of glycidyl azide polyurethane by NMR relaxation times. Macromolecules, 32:1602-1610.

[26] Vandenburg, E. J. (Hercules Inc.). 1972. U.S. Patent 3,645,917.

[27] Frankel, M. B., L. R. Grant, and J. E. Flanagan. 1992. Historical development of glycidyl azide polymer Journal of Propulsion and Power, 8(3):560-563. 
[28] Frankel, M. B., E. F. Witucki, and D.O. Woolery. 1983. U.S. Patent 4,379,894.

[29] Jutier, J-J., A. De Gunzborg, and R. E. Prud'Homme. 1999. Synthesis and characterization of poly(3,3 bis(azidomethyl)oxetane-co- $\varepsilon$-caprolactone)s. Journal of Polymer Science: Part A: Polymer Chemistry, 37:1027-1039.

[30] Zhai, J., R. Yang, and J. Li. 2008. Catalytic thermal decomposition and combustion of composite BAMO-THF propellants. Combustion and Flame, 154:473-477.

[31] Luo, Y., P. Chen, F-Q. Zhao, R-Z. Hu, S-W. Li, and Y. Gao. 2004. Kinetics and mechanism of the thermal decomposition reaction of 3,3bis(azidomethyl)oxetane/tetrahydrofuran copolymer. Chinese Journal of Chemistry, $22: 1219-1224$.

[32] Zhang Y., J. Zhao, P. Yang, S. He, and H. Huang. 2012. Synthesis and characterization of Energetic GAP-b-PAEMA block copolymer. Polym. Eng. Sci., $52: 768-773$.

[33] Manser, G. E., R. W. Fletcher, and G. C. Shaw. 1983. High Energy Binders.Contract No. N00014-82-C-0800, Morton Thiokol, Inc. Project JM101.

[34] Hardenstine, K. E., G. V. S. Henderson Jr, L. H. Sperling, C. J. Murphy, and G. E. Manser. 1985. Crystallization behavior of poly(3,3-bisethoxymethyl oxetane) and poly(3,3-bisazidomethyl oxetane)Journal of Polymer Science: Polymer Physics Edition, 23(8):1597-1609.

[35] Jones, R. B., C. J. Murphy, L. H. Sperling, M. Farber, S. P. Harris, and G. E. Manser. 1985. Thermal decomposition behavior of poly[3,3-bis(ethoxymethyl) oxetane] and related polyethers. Journal of Applied Polymer Science, 30(1):95-109.

[36] Bouchékif, H., M. I. Philbin, M. E. Colclough, and A. J. Amass. 2008. Cationic ringopening polymerization of oxetane via a non-steady-state controlled polymerization 
process: a comparison of initiators yielding living and nonliving polymers. Macromolecules, 41:1989-1995.

[37] Kawamoto, A. M., U. Barbieri, T. Keicher, H. Krause, J. A. S. Holanda, M. Kaiser, and G. Polacco. 2008. Synthesis and characterization of glycidyl azide-r-(3,3bis(azidomethyl)oxetane) copolymers. Propellant Explosives and Pyrotechnics, 33:365-372.

[38] Barbieri, U., T. Keicher, and G. Polacco. 2009. Homo and copolymers of 3tosyloxymethyl-3-methyl oxetane (TMMO) as precursors of energetic azide polymers. e-Polymers, 9(1):565-575.

[39] Pisharath, S., and H. G. Ang. 2007. Synthesis and thermal decomposition of GAP/Poly(BAMO) copolymer. Polymer Degradation and Stability, 92:1365-1377.

[40] Barbieri, U., G. Polacco, and R. Massimi. 2006. Synthesis of energetic polyethers from halogenated precursors. Macromolecular Symposia, 234(1):51-58.

[41] Barbieri, U., T. Keicher, R. Massimi, and G. Polacco. 2009. Preliminary Characterization of Propellants Based on GA/BAMO and pAMMO binders. Propellant Explosives and Pyrotechnics, 34(5):427-435.

[42] Kawamoto, A. M., M. F. Diniz, V. L. Lourenço, M. F. K. Takahashi, T. Keicher, H. Krause, K. Menke, and P. B. J. Kempa. 2010. Synthesis and characterization of GAP/BAMO copolymers applied at high energetic composite propellants. Aerosp. Technol. Manag., 2(3):307-322.

[43] Barbieri, U., G. Polacco, E. Paesano, and R. Massimi. 2006. Low risk synthesis of energetic poly(3-azidomethyl-3-methyloxetane) from tosylated precursors. Propellant Explosives and Pyrotechnics, 31(5):369-375. 
[44] Manu, S. K., T. L. Varghese, S. Mathe, and K. N. Ninan. 2009. Compatibility of glycidyl azide polymer with hydroxyl terminated polybutadiene and plasticizers. Journal of Propulsion and Power, 25(2):533-536.

[45] Mathew, S., S. K. Manu, and T. L. Varghese. 2008. Thermomechanical and morphological characteristics of cross-linked GAP and GAP-HTPB networks with different diisocyanates. Propellants, Explosives, Pyrotechnics, 33(2):146-152.

[46] Ding, Y., C. Hu, X. Guo, Y. Che, and J. Huang. 2014. Structure and mechanical properties of novel composites based on glycidyl azide polymer and propargylterminated polybutadiene as potential binder of solid propellant. Journal of Applied Polymer Science, 131(7): 40007.

[47] Hamley, I.W. 2004. Developments in block copolymer science and technology. Oxford UK, Oxford Univ Press, John Wiley and Sons.

[48] Zhou, Y., X-P. Long, and Q-X. J. Zeng. 2013. Simulation study of the morphologies of energetic block copolymers based on glycidyl azide polymer. Appl. Polym. Sci., $129: 480-486$.

[49] Zhou, Y., X-P. Long, and Q-X. J. Zeng. 2012. Simulation studies of the interfaces of incompatible glycidyl azide polymer/hydroxyl-terminated polybutadiene blends by dissipative particle dynamics. I. The effect of block copolymers and plasticizers. Appl. Polym. Sci., 125:1530-1537.

[50] Eroglu, M. S., B. Hazer, and o. Giiven. 1996. Synthesis and characterization of hydroxyl terminated poly(butadiene)-g-poly(glycidyl azide) copolymer as a new energetic propellant binder. Polymer Bulletin, 36:695-701.

[51] Mohan, Y. M., and K. M. Raju. 2005. Synthesis and characterization of HTPB-GAP cross-linked co-polymers. Designed Monomers and Polymers, 8(2):159-175. 
[52] Vasudevan, V., and G. Sundararajan 1999. Synthesis of GAP-PB-GAP triblock copolymer and application as modifier in AP/HTPB composite propellant. Propellants, Explosives, Pyrotechnics, 24:295-300.

[53] Subramanian K. 1999. Hydroxyl-terminated poly (azidomethyl ethylene oxide-bbutadiene-b-azidomethyl ethylene oxide) - synthesis, characterization and its potential as a propellant binder. European Polymer Journal, 35:1403-1411.

[54] Reddy, T. S., J. K. Nair, R. S. Satpute, R. M. Wagh, A. K. Sikder, and S. Venugopalan. 2007. Bis(azidomethyl) oxetane/hydroxyl-terminated polybutadiene/bis(azidomethyl) oxetane triblock copolymer: synthesis and characterization. Journal of Applied Polymer Science, 106:1885-1888.

[55] Nakabayashi, N., E. Masuhara, Y. Iwakura. 1966. Some reactions of the glycidyl esters of sulfonics acids. Bulletin of the Chemical Society of Japan, 39:413-417.

[56] Kazemi, F., A. R. Massah, M. Javaherian. 2007. Chemoselective and scalable preparation of alkyl tosylates under solvent-free conditions. Tetrahedron, 63:50835087.

[57] Selim, K., S.Özkar, and L. Yilmaz. 2000. Thermal characterization of glycidyl azide polymer (GAP) and GAP-based binders for composite propellants. Journal of Applied Polymer Science, 77:538-546.

[58] Kubota, N., and T. Sonobe. 1988. Combustion mechanism of azide polymer. Propellants, Explosives, Pyrotechnics, 13:172-177.

[59] Reshmia, S., K. P. Vijayalakshmi, Deepthi Thomas, B. K. George, and C. P. Reghunadhan Nair. 2013. Thermal decomposition of a diazido ester: Pyrolysis GCMS and DFT study. Journal of Analytical and Applied Pyrolysis, 104:603-608. 


\section{Figure captions}

Figure 1 Synthesis of the monomers. $\mathrm{G}=$ Glycidol; $\mathrm{GT}=$ glycidol tosylate; $\mathrm{HMMO}=3$ hydroxy-methyl-3-methyloxetane; TMMO = 3-tosyloxymethyl-3-methyl oxetane; BrBBrMP = 3-bromo-2,2-bis(bromomethyl)propanol; $\mathrm{BBrMO}=3,3$ bis(bromomethyl)oxetane.

Figure 2 Synthesis and azidation of the block copolymers.

Figure 3 H-NMR spectra of: (1) GT/BBrMO-PB-GT/BBrMO and (2) GA/BAMO-PBGA/BAMO from (1).

Figure 4 FT-IR spectra of: 1) GT/BBrMO-PB-GT/BBrMO; 2) GA/BAMO-PB-GA/BAMO from 1 ; 3) GA/BAMO-PB-GA/BAMO from 4; 4) ECH/BBrMO-PB$\mathrm{ECH} / \mathrm{BBrMO}$.

Figure 5 FT-IR spectra of: 5) GT-PB-GT; 6) GA/PB/GA; 7) TMMO-PB-TMMO; 8) AMMO-PB-AMMO.

Figure 6 GT-HTPB. Fluorescence microscopy of MB (a) and synthesis product (b); optical microscopy of MB (c) and synthesis product (d); chemical map of MB (e) and synthesis product (f). FTIR spectra corresponding to the positions indicated by the + symbols in (e) and (f).

Figure 7 GPC analysis of HTPB and GT-PB-GT copolymer. The dashed lines represent the deconvolution peaks of the GT-PB-GT distribution.

Figure 8 DSC traces of the first decomposition step for the azidated copolymers.

Figure 9 TGA analysis of HTPB, GA/BAMO-PB-GA/BAMO copolymers and their respective precursors.

Figure 10 TGA analysis of HTPB, GA-PB-GA, AMMO-PB-AMMO copolymers and their respective precursors. 
Table 1 - Composition of the reaction mixtures for the polymerizations.

\begin{tabular}{|c|c|c|c|c|c|c|c|c|}
\hline polymer & $\begin{array}{l}\text { HTPB } \\
\text { (g) } \\
\text { (mole) }\end{array}$ & $\begin{array}{c}\text { BBrMO } \\
\text { (g) } \\
\text { (mole) }\end{array}$ & $\begin{array}{c}\text { ECH } \\
(\mathrm{g}) \\
(\mathrm{mole})\end{array}$ & $\begin{array}{c}\text { GT } \\
\text { (g) } \\
\text { (mole) }\end{array}$ & $\begin{array}{c}\text { TMMO } \\
\text { (g) } \\
\text { (mole) }\end{array}$ & $\begin{array}{c}\text { BTF THF } \\
\text { (g) } \\
\text { (mole) }\end{array}$ & $\begin{array}{l}\text { DCM } \\
(\mathrm{mL})\end{array}$ & $\begin{array}{c}\mathrm{N}_{3} \\
(\mathrm{w} \%)\end{array}$ \\
\hline GT-PB-GT & $\begin{array}{c}1.5 \\
5.3 \cdot 10^{-4}\end{array}$ & - & - & $\begin{array}{c}13.65 \\
0.06\end{array}$ & - & $\begin{array}{c}0.336 \\
0.0024\end{array}$ & 110 & 35 \\
\hline GT/BBrMO-PB-GT/BBrMO & $\begin{array}{c}1.48 \\
5.3 \cdot 10^{-4}\end{array}$ & $\begin{array}{c}3.6 \\
0.015\end{array}$ & - & $\begin{array}{c}10 \\
0.044\end{array}$ & - & $\begin{array}{c}0.336 \\
0.0024\end{array}$ & 110 & 38 \\
\hline ECH/BBrMO-PB-ECH/BBrMO & 3.99 & 10.0 & 11.4 & - & - & $\begin{array}{c}0.91 \\
0.0065\end{array}$ & 110 & 38 \\
\hline TMMO-PB-TMMO & $\begin{array}{c}0.99 \\
3.5 \cdot 10^{-4}\end{array}$ & - & - & - & $\begin{array}{c}10.1 \\
0.039\end{array}$ & $\begin{array}{c}0.21 \\
0.0015\end{array}$ & 110 & 28 \\
\hline
\end{tabular}


Table 2 - Reaction time for the azidation of the copolymers.

\begin{tabular}{|l|c|c|c|c|}
\hline $\begin{array}{l}\text { monomeric } \\
\text { precursor }\end{array}$ & GT & GT/BBrMO & ECH/BBrMO & TMMO \\
\hline $\begin{array}{l}\text { Azidation } \\
\text { time (h) }\end{array}$ & 140 & 22 & 16 & 140 \\
\hline
\end{tabular}


Table 3. GPC results. $\mathrm{Mn}=$ number average molecular weight; $\mathrm{Mw}=$ weight average molecular weight.

\begin{tabular}{|l|l|l|l|l|l|l|l|}
\hline polymer & Mn & Mw & $\begin{array}{l}\text { Mn 1 peak } \\
(\mathrm{g} / \mathrm{mole})\end{array}$ & $\begin{array}{l}\text { Mw } 1^{\text {st }} \text { peak } \\
(\mathrm{g} / \mathrm{mole})\end{array}$ & $\begin{array}{l}\text { Mn 2 }{ }^{\text {nd }} \text { peak } \\
(\mathrm{g} / \mathrm{mole})\end{array}$ & $\begin{array}{l}\text { Mw 2 } \\
(\mathrm{g} / \mathrm{mole})\end{array}$ & $\begin{array}{l}\text { peak } \\
(\% \mathrm{gt})\end{array}$ \\
\hline HTPB & 4700 & 11400 & - & - & - & - & - \\
\hline GT-PB-GT & 1400 & 6230 & 1180 & 1340 & 6770 & 14850 & 21 \\
\hline ECH/BBrMO-PB-ECH/BBrMO & 610 & 13420 & 550 & 810 & 6650 & 15200 & 22 \\
\hline GT/BBrMO-PB-GT/BBrMO & 860 & 4990 & 750 & 1340 & 6930 & 14330 & 20 \\
\hline TMMO-PB-TMMO & 1850 & 9430 & 900 & 1020 & 7060 & 16200 & 15 \\
\hline
\end{tabular}


Table 4 - DSC data corresponding to the first decomposition step.

\begin{tabular}{|l|c|c|c|c|}
\hline Polymer & $\begin{array}{c}\mathrm{T}_{\mathrm{i}} \\
\left({ }^{\circ} \mathrm{C}\right)\end{array}$ & $\begin{array}{c}\mathrm{T}_{\mathrm{f}} \\
\left({ }^{\circ} \mathrm{C}\right)\end{array}$ & $\begin{array}{c}\Delta \mathrm{H} \\
(\mathrm{J} / \mathrm{g})\end{array}$ & $\begin{array}{c}\Delta \mathrm{H} \\
\left(\mathrm{J} / \mathrm{g} \mathrm{N}_{3}\right)\end{array}$ \\
\hline GA-PB-GA & 205 & 270 & 1,380 & 3,940 \\
\hline $\begin{array}{l}\text { GA/BAMO-PB-GA/BAMO } \\
\text { from } \\
\text { ECH/BBrMO-PB-ECH/BBrMO }\end{array}$ & 175 & 240 & 1,740 & 4,575 \\
\hline $\begin{array}{l}\text { GA/BAMO-PB-GA/BAMO } \\
\text { from } \\
\text { GT/BBrMO-PB-GT/BBrMO }\end{array}$ & 210 & 270 & 1,875 & 4,940 \\
\hline AMMO-PB-AMMO & 175 & 255 & 630 & 2,250 \\
\hline
\end{tabular}


Table 5 - TGA data for energetic polymers and corresponding precursors.

\begin{tabular}{|l|c|c|c|c|}
\hline \multirow{2}{*}{ Polymer } & \multicolumn{3}{|c|}{$\mathrm{N}_{3}$ degradation } & \multirow{2}{*}{$\mathrm{W}_{\mathrm{r}}$} \\
\cline { 2 - 5 } & $\begin{array}{c}\mathrm{T}_{\mathrm{i}}-\mathrm{T}_{\mathrm{f}} \\
\left({ }^{\circ} \mathrm{C}\right)\end{array}$ & $\begin{array}{c}\Delta \mathrm{w} \\
(\%)\end{array}$ & $\begin{array}{c}\Delta \mathrm{w} \mathrm{N}_{3} \\
(\%)\end{array}$ & \\
\hline HTPB & & & & 0.5 \\
\hline GT-PB-GT & $210-280$ & 47 & 201 & 15 \\
\hline GA-PB-GA & - & - & & 2.2 \\
\hline ECH/BBrMO-PB-ECH/BBrMO & $175-275$ & 41.2 & 164 & 24.1 \\
\hline $\begin{array}{l}\text { GA/BAMO-PB-GA/BAMO } \\
\text { from }\end{array}$ & & & & 6.1 \\
\hline ECH/BBrMO-PB-ECH/BBrMO & $200-275$ & 43.8 & 175 & 25.6 \\
\hline $\begin{array}{l}\text { GT/BBrMO-PB-GT/BBrMO } \\
\text { from } \\
\text { GT/BBMOMO-PB-GT/BBrMO }\end{array}$ & & & & 6.3 \\
\hline TMMO-PB-TMMO & $159-250$ & 12.5 & 70 & 4.2 \\
\hline AMMO-PB-AMMO & & & & \\
\hline
\end{tabular}

22 
<smiles>OCC(CBr)(CBr)CBr</smiles>
BrBBrMP

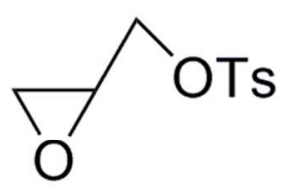

GT

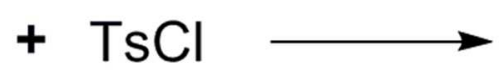

HMMO

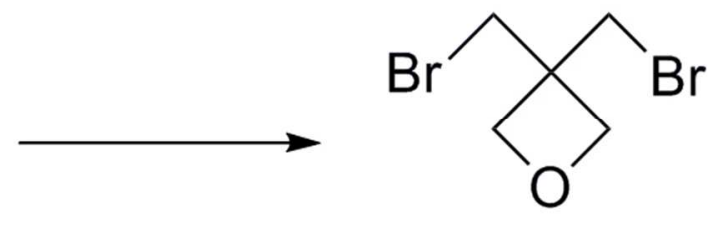

Figure 1 - Synthesis of the monomers. G = Glycidol; GT = glycidol tosylate; HMMO = 3-hydroxy-methyl-3methyloxetane; TMMO = 3-tosyloxymethyl-3-methyl oxetane; BrBBrMP = 3-bromo-2,2bis(bromomethyl)propanol; BBrMO = 3,3-bis(bromomethyl)oxetane. $81 \times 76 \mathrm{~mm}(300 \times 300 \mathrm{DPI})$ 
Figure 2 - Synthesis and azidation of the block copolymers. $272 \times 183 \mathrm{~mm}(300 \times 300 \mathrm{DPI})$ 


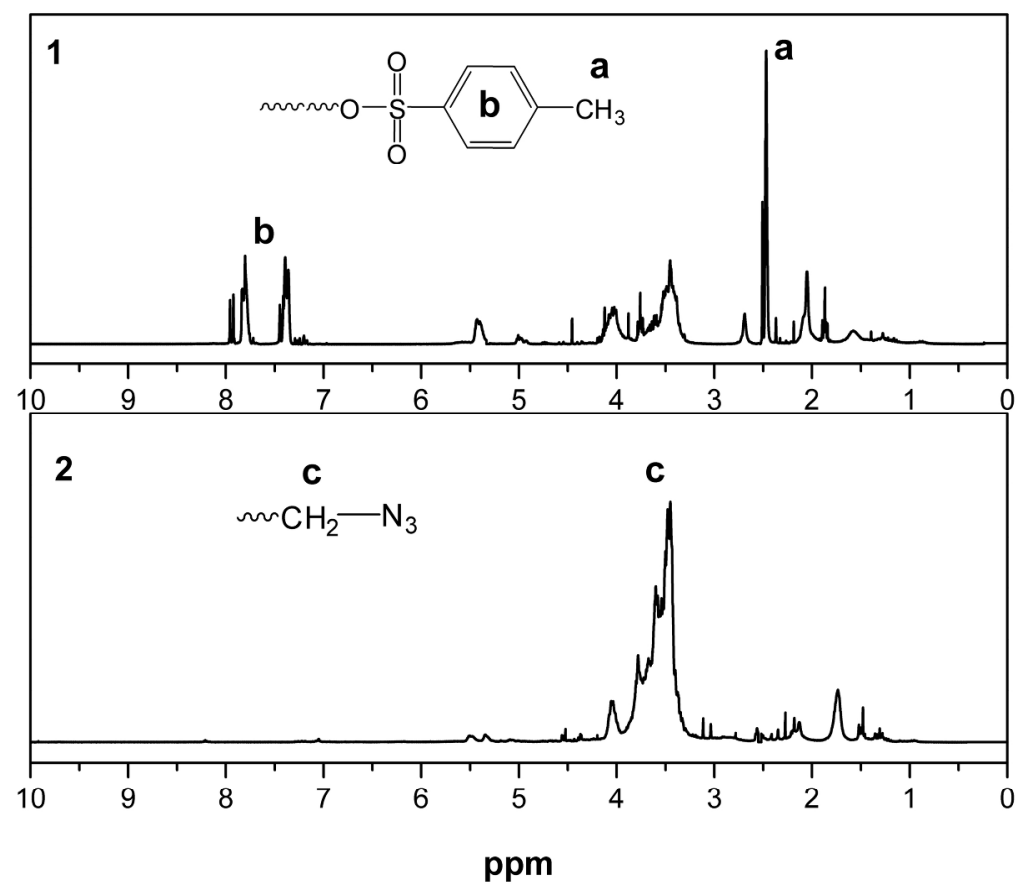

Figure 3 - H-NMR spectra of: (1) GT/BBrMO-PB-GT/BBrMO and (2) GA/BAMO-PB-GA/BAMO from (1). $227 \times 174 \mathrm{~mm}(300 \times 300 \mathrm{DPI})$ 


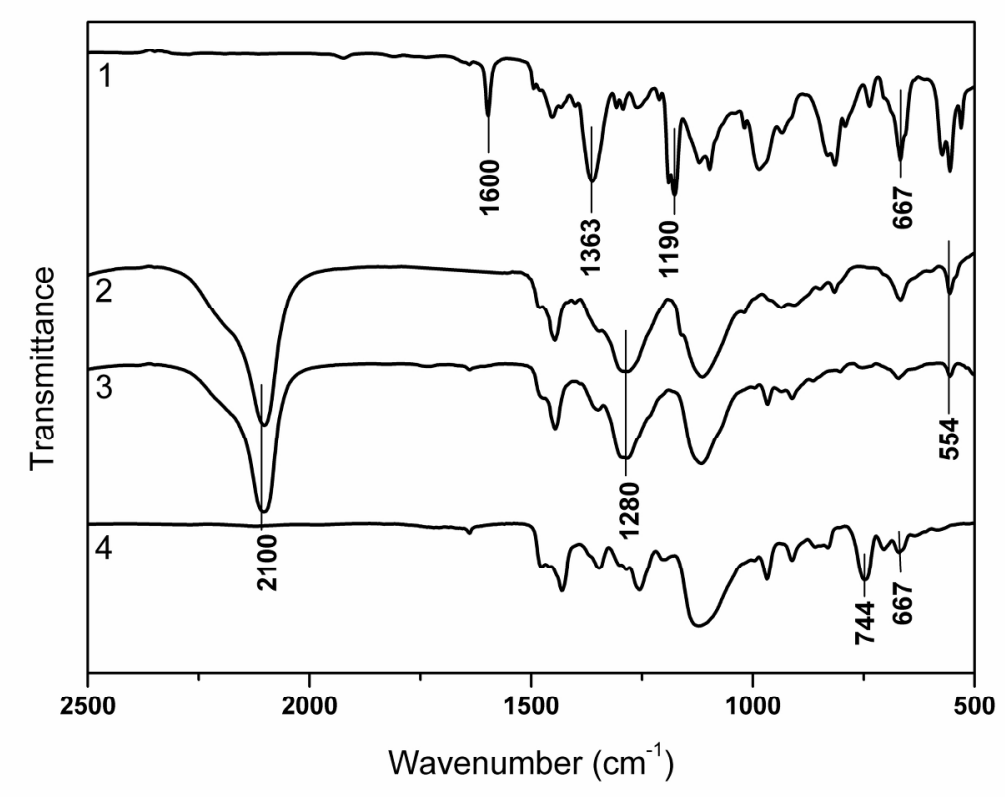

Figure 4 - FT-IR spectra of: 1) GT/BBrMO-PB-GT/BBrMO; 2) GA/BAMO-PB-GA/BAMO from 1; 3) GA/BAMOPB-GA/BAMO from 4; 4) ECH/BBrMO-PB-ECH/BBrMO. $207 \times 144 \mathrm{~mm}(300 \times 300 \mathrm{DPI})$ 


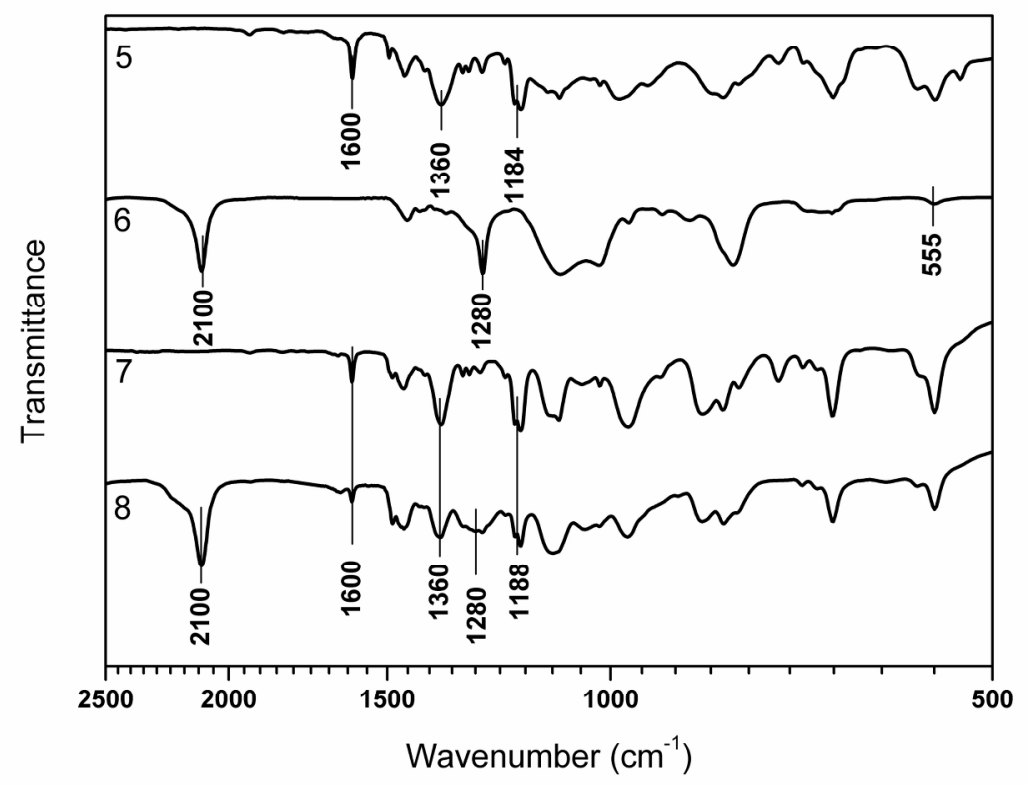

Figure 5 - FT-IR spectra of: 5) GT-PB-GT; 6) GA/PB/GA; 7) TMMO-PB-TMMO; 8) AMMO-PB-AMMO. $207 \times 144 \mathrm{~mm}(300 \times 300 \mathrm{DPI})$ 

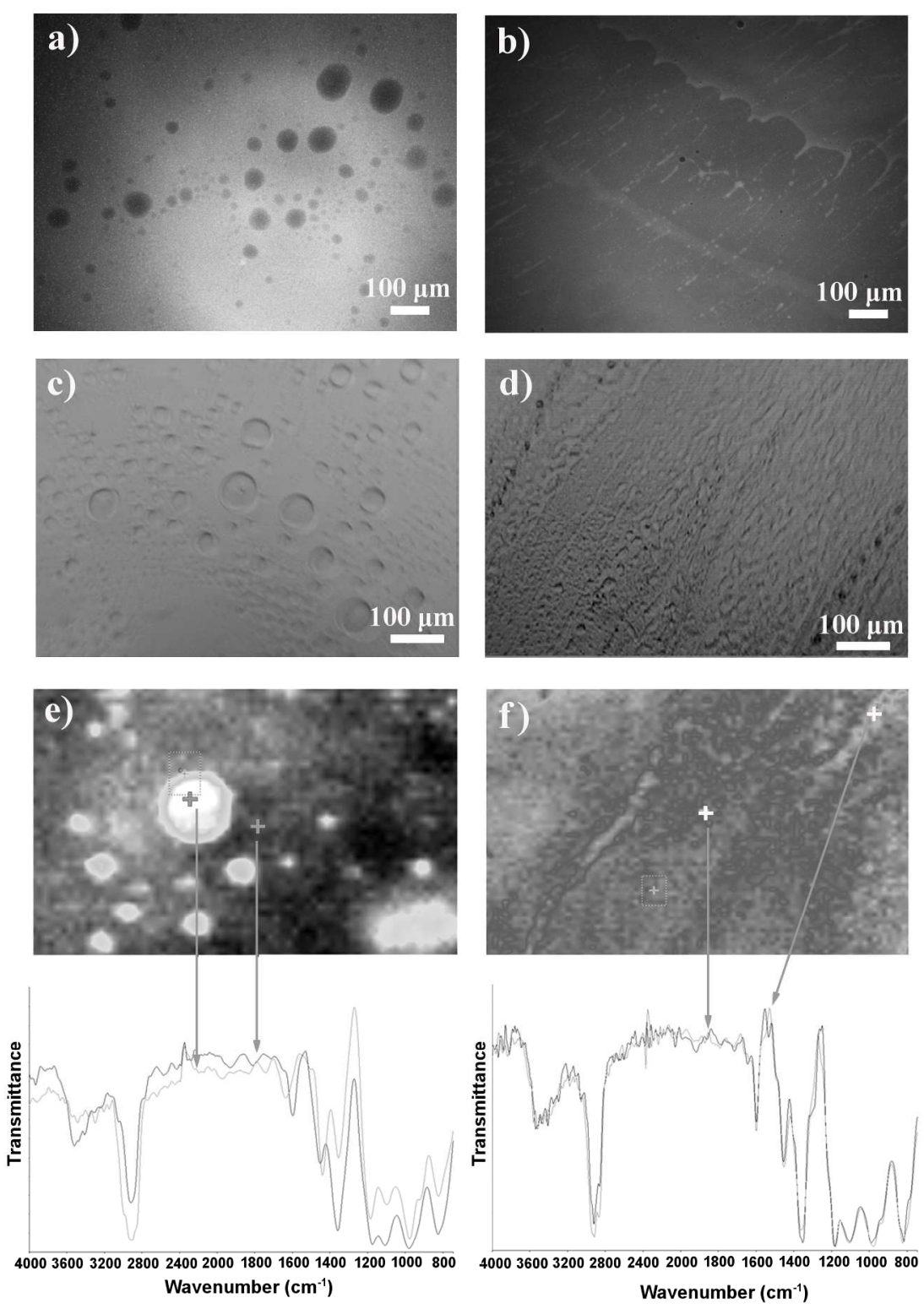

Figure 6 GT-HTPB. Fluorescence microscopy of MB (a) and synthesis product (b); optical microscopy of MB

(c) and synthesis product (d); chemical map of MB (e) and synthesis product (f). FTIR spectra corresponding to the positions indicated by the + symbols in (e) and (f). $175 \times 249 \mathrm{~mm}(300 \times 300 \mathrm{DPI})$ 


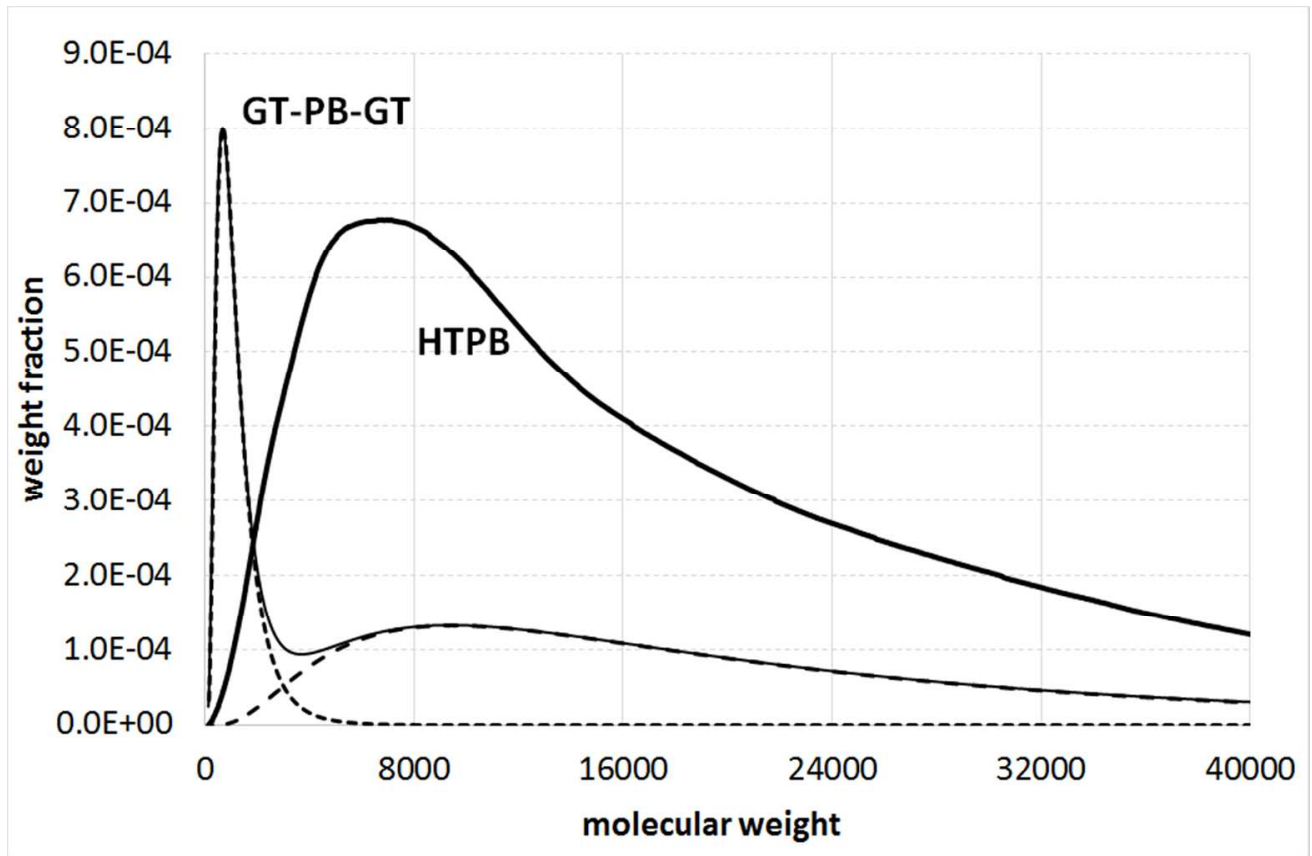

Figure 7 GPC analysis of HTPB and GT-PB-GT copolymer. The dashed lines represent the deconvolution peaks of the GT-PB-GT distribution. 
Figure 8 DSC traces of the first decomposition step for the azidated copolymers. $210 \times 148 \mathrm{~mm}(300 \times 300 \mathrm{DPI})$ 


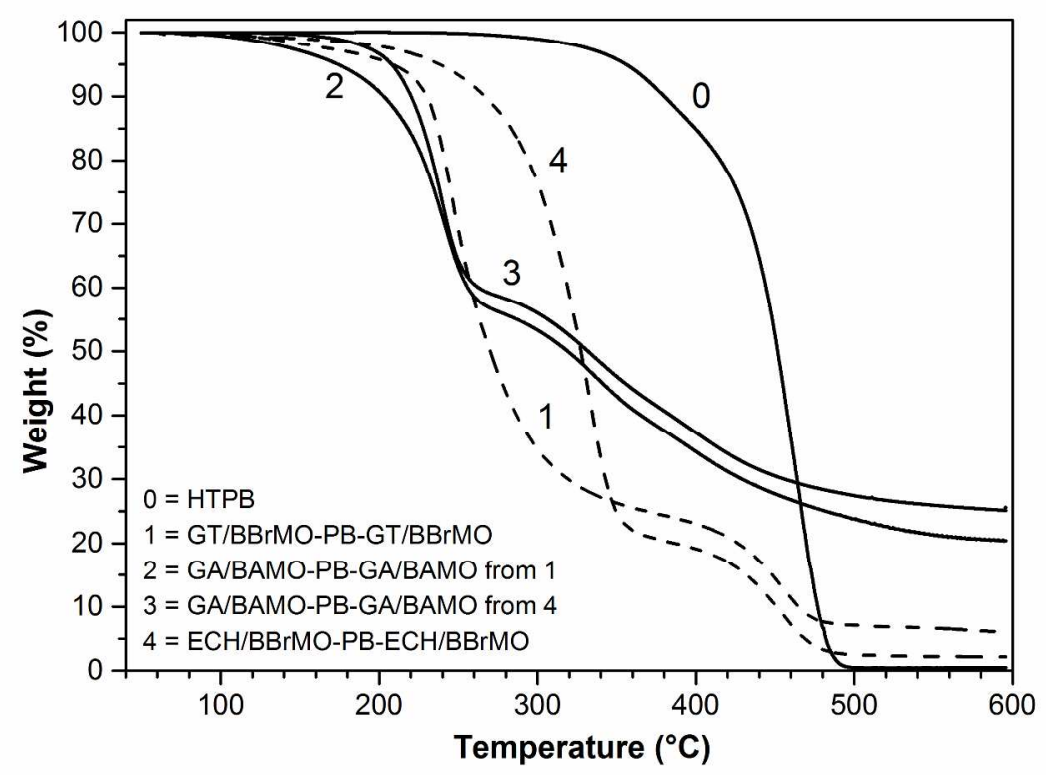

Figure 9 TGA analysis of HTPB, GA/BAMO-PB-GA/BAMO copolymers and their respective precursors. $286 \times 199 \mathrm{~mm}(300 \times 300 \mathrm{DPI})$ 


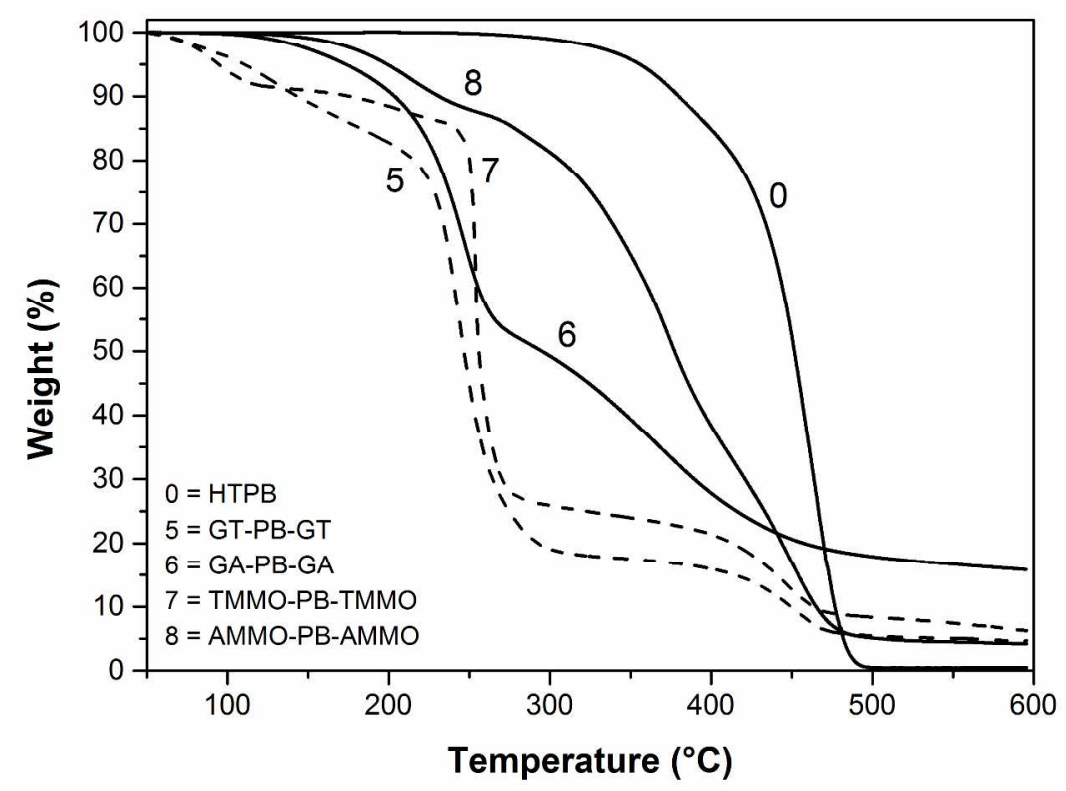

Figure 10 TGA analysis of HTPB, GA-PB-GA, AMMO-PB-AMMO copolymers and their respective precursors. $286 \times 199 \mathrm{~mm}(300 \times 300 \mathrm{DPI})$ 